\title{
Publisher Correction: Direct quantitative material decomposition employing grating- based X-ray phase-contrast CT
}

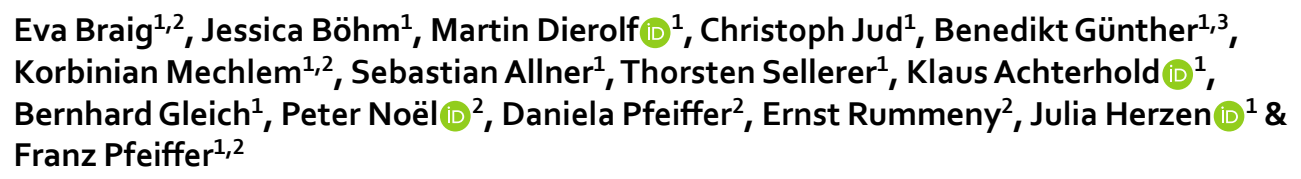

Correction to: Scientific Reports https://doi.org/10.1038/s41598-018-34809-6, published online 06 November 2018

This Article contains typesetting errors.

In the Materials and Methods section, under the subheading 'Munich Compact Light Source',

"The spectrum at the position of the sample was measured with an energy-dispersive Amptek X-123 detector (Amptek Inc., Bedford, Massachusetts) with an $500 \mathrm{~m} \mathrm{Si}$ sensor and from that a mean energy of $E_{\text {mean }}=24.3 \mathrm{keV}$ was calculated."

should read:

"The spectrum at the position of the sample was measured with an energy-dispersive Amptek X-123 detector (Amptek Inc., Bedford, Massachusetts) with an $500 \mu \mathrm{m} \mathrm{Si} \mathrm{sensor} \mathrm{and} \mathrm{from} \mathrm{that} \mathrm{a} \mathrm{mean} \mathrm{energy} \mathrm{of} E_{\text {mean }}=24.3 \mathrm{keV}$ was calculated."

Furthermore, in the Materials and Methods section, under the subheading 'Grating Interferometer',

"The grating interferometer is situated at a distance of about $15 \mathrm{~m}$ from the X-ray source point (size: $45 \times 45 \mathrm{~m}^{2}$ r.m.s., divergence angle: $4 \mathrm{mrad)}$."

should read:

"The grating interferometer is situated at a distance of about $15 \mathrm{~m}$ from the X-ray source point (size: $45 \times 45 \mu \mathrm{m}^{2}$ r.m.s., divergence angle: $4 \mathrm{mrad})$."

"The Talbot interferometer is realized with a phase grating (G1) with a period of $4.92 \mathrm{~m}$, duty cycle of 0.5 and a nickel filling height of $4.39 \mathrm{~m}$ providing a phase shift of $\pi / 2$ for the design energy of $25 \mathrm{keV}$. Period, duty cycle and gold filling for the absorption grating are $\mathrm{p}_{2}=5 \mathrm{~m}, 0.5$ and $70 \mathrm{~m}$."

should read:

\footnotetext{
${ }^{1}$ Chair of Biomedical Physics, Department of Physics and Munich School of BioEngineering, Technical University of Munich, 85748, Garching, Germany. ${ }^{2}$ Department of Diagnostic and Interventional Radiology, Klinikum rechts der Isar, Technical University of Munich, 81675, München, Germany. ${ }^{3}$ Max-Planck-Institute of Quantum Optics, HansKopfermann-Straße 1, 85748, Garching, Germany. Correspondence and requests for materials should be addressed to E.B. (email: eva.braig@mytum.de)
} 
"The Talbot interferometer is realized with a phase grating (G1) with a period of $4.92 \mu \mathrm{m}$, duty cycle of 0.5 and a nickel filling height of $4.39 \mu \mathrm{m}$ providing a phase shift of $\pi / 2$ for the design energy of $25 \mathrm{keV}$. Period, duty cycle and gold filling for the absorption grating are $\mathrm{p}_{2}=5 \mu \mathrm{m}, 0.5$ and $70 \mu \mathrm{m}$.”

"All tomographic images were acquired with a single photon counting Pilatus-200 K detector (DECTRIS ltd., Baden, Switzerland) with a $1 \mathrm{~mm}$ thick silicon sensor and an effective pixel size of $p_{\text {eff }}=160 \mathrm{~m}$."

should read:

"All tomographic images were acquired with a single photon counting Pilatus-200 K detector (DECTRIS ltd., Baden, Switzerland) with a $1 \mathrm{~mm}$ thick silicon sensor and an effective pixel size of $p_{\text {eff }}=160 \mu \mathrm{m}$."

(c) (i) Open Access This article is licensed under a Creative Commons Attribution 4.0 International License, which permits use, sharing, adaptation, distribution and reproduction in any medium or format, as long as you give appropriate credit to the original author(s) and the source, provide a link to the Creative Commons license, and indicate if changes were made. The images or other third party material in this article are included in the article's Creative Commons license, unless indicated otherwise in a credit line to the material. If material is not included in the article's Creative Commons license and your intended use is not permitted by statutory regulation or exceeds the permitted use, you will need to obtain permission directly from the copyright holder. To view a copy of this license, visit http://creativecommons.org/licenses/by/4.0/.

(C) The Author(s) 2019 